\title{
Efeito de extratos aquosos de Serjania spp. sobre a oviposição de Plutella xylostella L. (Lepidoptera: Plutellidae)
}

Effect of aqueous extracts of Serjania spp. on the oviposition of Plutella xylostella L. (Lepidoptera: Plutellidae)

Efecto de extractos acuosos de Serjania spp. sobre la oviposición de Plutella xylostella L. (Lepidoptera: Plutellidae)

Recebido: 14/10/2021 | Revisado: 21/10/2021 | Aceito: 27/10/2021 | Publicado: 30/10/2021

Eduardo Carvalho Faca

ORCID: https://orcid.org/0000-0002-2718-1871 Universidade Federal da Grande Dourados, Brasil

E-mail: eduardofaca@gmail.com

Eliana Aparecida Ferreira

ORCID: https://orcid.org/0000-0002-4254-7050

Universidade Federal da Grande Dourados, Brasil E-mail: lih.ferreira.ivi@gmail.com

Rosicleia Matias da Silva

ORCID: https://orcid.org/0000-0001-8988-1948 Universidade Federal da Grande Dourados, Brasil E-mail: rosi-matias09@hotmail.com

Rosilda Mara Mussury

ORCID: https://orcid.org/0000-0002-8961-9146 Universidade Federal da Grande Dourados, Brasil E-mail: mussuryufgd@gmail.com

\begin{abstract}
Resumo
A bioprospecção de espécies vegetais para o desenvolvimento de bioinsetidas pode contribuir para o controle de populações de Plutella xylostella na cultura das brássicas. O objetivo desta pesquisa foi estudar o efeito dos extratos aquosos de Serjania erecta e Serjania marginata, nas concentrações 5 e 10\%, sobre a oviposição de P. xylostella. Para isso, foram realizados dois testes: o primeiro consistiu em um teste sem chance de escolha, onde foi colocado em cada gaiola um disco de couve tratado e um casal de adultos de $P$. xylostella; o segundo consistiu em um teste com chance de escolha, onde foram colocados em cada gaiola quatro discos tratados (dois discos tratados com extrato vegetal e dois discos tratados com água destilada) e um casal de adultos de $P$. xylostella. O experimento de oviposição sem chance de escolha foi realizado em delineamento experimental inteiramente casualizado (DIC) em esquema fatorial duplo ( $3 \times 2)$ com 15 repetições por tratamento e o experimento com chance de escolha em delineamento experimental inteiramente casualizado (DIC). Foram contabilizados, diariamente, o número de ovos (fecundidade) e o número de larvas recémemergidas (fertilidade dos ovos) durante 10 dias e calculado a viabilidade dos ovos (\%) e o Índice de Dissuasão de Oviposição (IDO). Os resultados indicaram o efeito dissuasor das duas espécies vegetais, com redução da fecundidade, fertilidade e viabilidade de ovos nos tratamentos com os extratos aquosos de $S$. erecta e $S$. marginata, nas concentrações $5 \%$ e $10 \%$, sendo que a concentração $10 \%$ apresentou melhores resultados para o teste sem chance de escolha. Portanto, as duas espécies vegetais apresentaram ação inseticida, atuando na redução da fecundidade, fertilidade, viabilidade de ovos e na dissuasão de oviposição deste microlepidóptero, contribuindo para o seu controle em cultivos de hortaliças. Palavras-chave: Bioinseticidas; Dissuasão; Extratos botânicos; Sapindaceae; Traça-das-crucíferas.
\end{abstract}

\begin{abstract}
The bioprospecting of plant species for the development of bioinsecticides can contribute to the control of Plutella xylostella populations in the brassica culture. The objective of this research was to study the effect of the aqueous extracts of Serjania erecta and Serjania marginata, at concentrations 5 and 10\%, on the oviposition of P. xylostella. For this, two tests were performed: the first consisted of a no-choice test, in which a treated cabbage disk and a pair of $P$. xylostella adults were placed in each cage; the second consisted of a free-choice test, where four treated discs (two discs treated with plant extract and two discs treated with distilled water) and a pair of adults of P.xylostella were placed in each cage. The no-choice oviposition experiment was carried out in a completely randomized experimental design (DIC) in a double factorial scheme ( $3 \times 2)$ with 15 replications per treatment and the no-choice experiment was carried out in a completely randomized experimental design (DIC). The number of eggs (fecundity) and the number of newly
\end{abstract}


emerged larvae (fertility of eggs) were counted daily for 10 days and the egg viability (\%) and the Oviposition Dissuasion Index (ODI) were calculated. The results indicated the deterrent effect of the two plant species, with reduced fecundity, fertility and egg viability in treatments with aqueous extracts of S. erecta and $S$. marginata, at concentrations of $5 \%$ and $10 \%$, with a concentration of $10 \%$ presented better results for the no-choice test. Therefore, the two plant species presented insecticidal action, acting in the reduction of fecundity, fertility, egg viability and in the deterrence of oviposition of this microlepidoptera, contributing to its control in vegetable crops.

Keywords: Biopesticides; Botanical extracts; Cruciferous moth; Dissuasion; Sapindaceae.

\section{Resumen}

La bioprospección de especies vegetales para el desarrollo de bioinsecticidas puede contribuir al control de las poblaciones de Plutella xylostella en el cultivo de Brassica. El objetivo de esta investigación fue estudiar el efecto de los extractos acuosos de Serjania erecta y Serjania marginata, a concentraciones de 5 y 10\%, sobre la oviposición de P. xylostella. Para ello se realizaron dos pruebas: la primera consistió en una prueba de no elección, en la que se colocaron en cada jaula un disco de repollo tratado y una pareja de adultos de P. xylostella; el segundo consistió en una prueba de libre elección, donde se colocaron cuatro discos tratados (dos discos tratados con ex tracto de planta y dos discos tratados con agua destilada) y un par de adultos de $P$. xylostella en cada jaula. El experimento de oviposición sin elección se realizó en un diseño experimental completamente aleatorizado (DIC) en un esquema factorial doble (3x2) con 15 repeticiones por tratamiento y el experimento sin elección se realizó en un diseño experimental completamente aleatorizado (DIC). El número de huevos (fecundidad) y el número de larvas recién emergidas (fertilidad de huevos) se contaron diariamente durante 10 días y se calculó la viabilidad del huevo (\%) y el Índice de Disuasión de Oviposición (ODI). Los resultados indicaron el efecto disuasorio de las dos especies vegetales, con reducción de fecundidad, fertilidad y viabilidad de huevos en tratamientos con extractos acuosos de $S$. erecta y $S$. marginata, a concentraciones de $5 \%$ y $10 \%$, con una concentración de $10 \%$ presentada mejores resultados para la prueba de no elección. Por tanto, las dos especies vegetales presentaron acción insecticida, actuando en la reducción de la fecundidad, fertilidad, viabilidad del huevo y en la disuasión de la oviposición de este microlepidóptero, contribuyendo a su control en cultivos hortícolas.

Palabras clave: Bioplaguicidas; Disuasión; Extractos botánicos; Polilla crucífera; Sapindaceae.

\section{Introdução}

A traça-das-crucíferas, Plutella xylostella L. (Lepidoptera: Plutellidae), é uma das principais pragas que se alimenta de brássicas e que causa importantes danos econômicos nesta cultura (Zalucki et al., 2012; Massarolli et al., 2019). Devido a sua alta capacidade reprodutiva e ao seu curto ciclo de vida, esse microlepidópero pode permanecer na cultura das brássicas por várias gerações, causando grandes prejuízos econômicos (Ulmer et al., 2002; Marchioro \& Foerster, 2011) e exigindo grandes investimentos em inseticidas sintéticos, sendo que o custo anual mundial relacionado ao seu controle pode chegar a US $\$ 5$ bilhões (Zalucki et al., 2012).

A utilização de inseticidas sintéticos no manejo de $P$. xylostella é o método mais utilizado, pois garante rápido controle das populações na cultura (Agboyi et al., 2016), porém, a utilização incorreta deste recurso tornou este inseto resistente a mais de 101 princípios ativos de agrotóxicos registrados mundialmente (APRD, 2021). Entretanto, estes produtos, na maioria das vezes, não são seletivos e podem comprometer a entomofauna não alvo e benéfica, além de gerar resíduos no ambiente e propiciar o desenvolvimento de resistência do inseto alvo (Furlong et al., 2004; Li et al., 2012; Zhang, et al., 2016). Algumas pesquisas evidenciam que os inseticidas sintéticos podem afetar organismos não alvos em solos e em ambientes aquáticos, justificando a importância dos estudos ecotoxicológicos nos diferentes nichos ecológicos (Iannacone et al., 2007, 2008).

Diante das desvantagens e do alto custo da utilização de inseticidas sintéticos, a utilização alternativa ou complementar de inseticidas botânicos é uma saída viável quando associada a outras estratégias do Manejo Integrado de Pragas (MIP) (Loiseleur, 2017). Os inseticidas botânicos apresentam vantagens e praticidades, como a utilização de plantas nativas, baixo impacto ambiental, além do baixo custo, sendo assim, vantajoso principalmente para os pequenos produtores (Amoabeng et al., 2013; Pavela, 2016). A prospecção de espécies botânicas para o desenvolvimento de estudos que promovam a investigação de bioatividade vegetal com ênfase na ação inseticida tem aumentado ao longo dos anos (Isman, 2014; Peres et al., 2017; Fonseca 
et al., 2018; Couto et al., 2019, 2020; Souza et al., 2019; Ferreira et al., 2020). Alguns trabalhos demonstram que os inseticidas botânicos podem inibir e reduzir a alimentação e reprodução de insetos (Couto et al., 2019), reduzir a fecundidade (Couto et al., 2020), interferir na síntese de ecdisona e causar esterilidade (Schmutterer et al., 1988; Mordue \& Blackwell 1993), além de provocar deformações em pupas e adultos e ocasionar mortalidade de indivíduos imaturos e adultos (Peres et al., 2017).

A ação inseticida é gerada a partir de substâncias bioativas oriundas do metabólito secundários de determinadas espécies vegetais, sendo um mecanismo de defesa contra o ataque de agentes agressores (Tagliari et al., 2001). Estas substâncias bioativas podem agir como inseticidas e são compartilhadas por diversas famílias botânicas como Annonaceae, Solanaceae, Meliaceae e Sapindaceae, sendo relatadas em vários estudos (Castilho-Sánchez et al., 2010; Krinski et al., 2014; Couto et al., 2020).

Dentre as famílias vegetais, a Sapindaceae é investigada em alguns estudos apresentando espécies com potencial ação inseticida (Couto et al., 2019; 2020; Ramírez-Zamora et al., 2020). Os compostos mais abundantes encontrados nessa família são os compostos fenólicos, flavonoides, esteroides, taninos, saponinas e antraquinonas, que podem apresentar ação inseticida para determinados insetos (Quintanilla et al., 2014; Guimarães et al., 2015; Moreira et al., 2019 Salinas-Sánchez et al., 2020). Diante da necessidade de minimizar as aplicações de inseticidas sintéticos e seus impactos ao meio ambiente, bem como estimular o desenvolvimento de novos bioinseticidas para controlar as populações de $P$. xylostella no campo, e atender tanto o mercado mundial como o local, avaliamos o potencial efeito de extratos aquosos de Serjania erecta e $S$. marginata nas concentrações $5 \%$ e $10 \%$ sobre a reprodução e oviposição de P. xylostella.

\section{Metodologia}

Os extratos e os bioensaios foram conduzidos e preparados no Laboratório de Interação Inseto-Planta (LIIP) da Faculdade de Ciências Biológicas e Ambientais na Universidade Federal da Grande Dourados - UFGD, Dourados, Mato Grosso do Sul, Brasil.

\subsection{Criação de $P$. xylostella}

A criação foi estabelecida e multiplicada sob condições controladas de temperatura $\left(25 \pm 2{ }^{\circ} \mathrm{C}\right)$, umidade relativa $(55 \pm$ $5 \%$ e fotoperíodo (12 horas), a partir de pupas e lagartas coletadas em áreas de cultivo de couve orgânica no município de Dourados - MS.

As pupas foram colocadas em gaiolas transparentes de plástico $(9 \mathrm{~cm}$ de comprimento x $19 \mathrm{~cm}$ de largura x $19 \mathrm{~cm}$ de altura) até a emergência dos adultos, que foram alimentados com mel a 10\%. Foram oferecidos discos de couve ( $8 \mathrm{~cm}$ de diâmetro) sobre papel filtro para a oviposição das fêmeas. Os discos contendo as posturas foram substituídos diariamente e transferidos para potes de plástico (30 cm de comprimento $\times 15 \mathrm{~cm}$ de largura $\times 12 \mathrm{~cm}$ de altura), devidamente esterilizados. Após a emergência das larvas, estas foram alimentadas com folhas de couve orgânica (Brassica oleracea var. acephala), higienizadas e lavadas com solução de hipoclorito de sódio a 5\% e água corrente. As folhas de couve foram dispostas com face adaxial voltada para o recipiente de plástico e a face abaxial livre, onde foram colocadas as larvas. Em seguida foi introduzida outra folha com a face abaxial voltada para as larvas. As folhas de couve foram substituídas diariamente, mantendo sempre as folhas superiores e as lagartas permaneceram nos potes até atingirem a fase pupal (Barros et al., 2012) (Figura 1). 
Figura 1. Esquema representativo da metodologia de criação e multiplicação de $P$. xylostella em condições controladas de temperatura $\left(25 \pm 2{ }^{\circ} \mathrm{C}\right)$, umidade relativa $(55 \pm 5 \%)$ e fotoperíodo (12 horas).

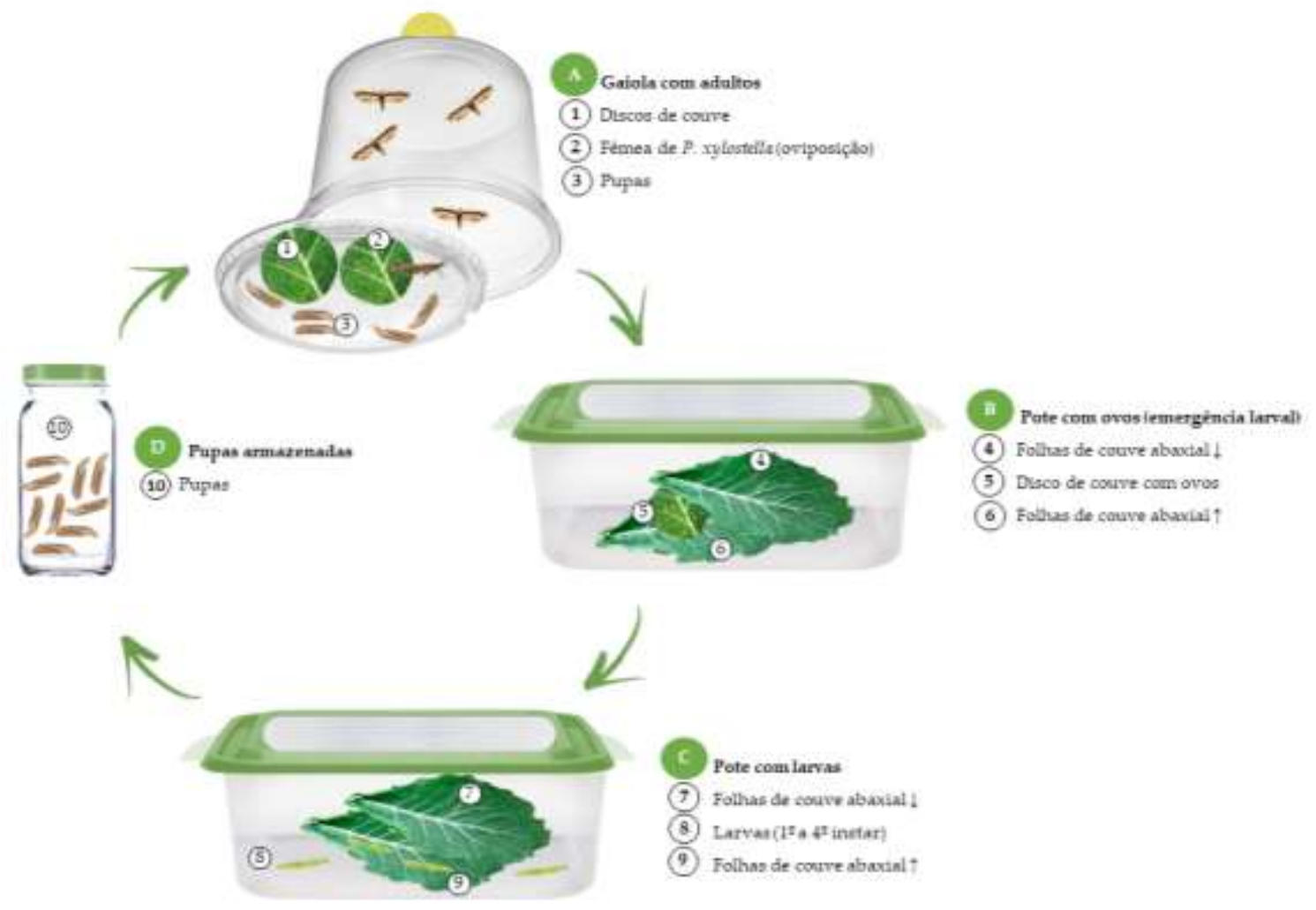

Fonte: Autores.

\subsection{Material botânico}

Folhas expandidas de S. erecta e S. marginata foram coletadas no mês de agosto, no período vespertino, no Horto de

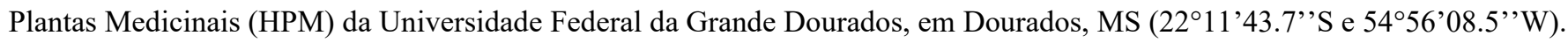

As espécies foram identificadas a partir das exsicatas presentes no herbário da Universidade Federal da Grande Dourados-UFGD, com os respectivos números de registro: 5395 - S. erecta e 6267 - S. marginata.

\subsection{Preparo dos extratos aquosos}

As folhas coletadas foram colocadas em câmara de circulação forçada de ar com temperatura constante de $40{ }^{\circ} \mathrm{C}( \pm 1$ ${ }^{\circ} \mathrm{C}$ ), durante 3 dias. Em seguida, as folhas foram trituradas em moinho industrial de facas até a obtenção de um pó fino.

A técnica de maceração foi utilizada para a preparação do extrato aquoso, onde misturou-se $5 \mathrm{~g}$ e 2,5 g do material vegetal com $50 \mathrm{~mL}$ de água destilada para obtenção das concentrações de $10 \%$ e 5\%, respectivamente. Depois da agitação manual, este material permaneceu em repouso por 24 horas sob refrigeração para a extração dos compostos hidrossolúveis. Após este período foi realizada a coagem, com o auxílio de tecido voil, para a obtenção dos extratos nas concentrações (peso/volume) de $10 \%$ e $5 \%$.

\subsection{Bioensaios de oviposição com extratos aquosos em $P$. xylostella}

Para os testes de oviposição sem chance e com chance de escolha foram selecionados casais de adultos de P. xylostella 
com até 12 horas de idade, procedentes da criação do laboratório. Discos foliares de couve foram imersos nos tratamentos (2 espécies vegetais, 2 concentrações e 1 controle) e após secarem naturalmente foram colocados em gaiolas plásticas. Os casais de P. xylostela foram mantidos por dez dias nas gaiolas e alimentados com solução de mel a 10\%. Os discos foram substituídos diariamente, e com auxílio do microscópio estereoscópico foram avaliados os ovos. Os bioensaios foram conduzidos e executados em condições controladas em laboratório $\left(25 \pm 1{ }^{\circ} \mathrm{C}, 55 \pm 5 \%\right.$ de UR e fotoperíodo de 12 horas) (Figura 2).

\subsubsection{Bioensaio de oviposição sem chance de escolha}

Para o bioensaio de oviposição sem chance de escolha foi disponibilizado, em cada gaiola, um único tratamento, ou seja, um disco foliar de couve devidamente tratado com água destilada, e os respectivos extratos aquosos ( S. erecta $5 \%$, S. erecta $10 \%$, S. marginata $5 \%$ ou S. marginata $10 \%$ ), com um casal de P. xylostella em cada gaiola. Neste bioensaio foram utilizados 15 casais de $P$. xylostella, totalizando 15 gaiolas para cada tratamento. Foram avaliados a fecundidade (número total de ovos), a fertilidade dos ovos (número total de larvas), a viabilidade dos ovos (\%) (porcentagem de ovos viáveis) e o Índice de Dissuasão de Oviposição (IDO) (Figura 2).

O cálculo do índice de dissuasão foi determinado pelo número de ovos do tratamento controle e pelo número de ovos do tratamento com extrato, com base na formula: $\mathrm{IDO}=(\mathrm{C}-\mathrm{T}) /(\mathrm{C}+\mathrm{T}) \times 100$, em que, $\mathrm{C}$ é igual ao controle, e $\mathrm{T}$ é igual ao tratamento, classificado como dissuasor se o índice for maior que zero; neutro se o índice for igual a zero; e estimulante, se o índice for menor que zero (Lundgren, 1975; Huang \& Renwick, 1993).

$\mathrm{O}$ experimento de oviposição sem chance de escolha foi realizado em delineamento experimental inteiramente casualizado (DIC) em esquema fatorial duplo (3x2), sendo duas espécies de plantas, um controle e duas concentrações de extrato vegetal $(5 \%$ e $10 \%)$. Os resultados foram submetidos à análise de variância e as médias comparadas pelo teste de Tukey ( $\mathrm{p}<$ $0,05)$ (Figura 1).

\subsubsection{Bioensaio de oviposição com chance de escolha}

Para o bioensaio de oviposição com chance de escolha foram dispostos na mesma placa, quatro discos de couve (2 discos tratados com extrato vegetal das espécies estudadas e concentrações testadas e 2 tratados com água destilada), de forma equidistante, e, em cada gaiola, um casal de adultos de P. xylostella. Neste bioensaio foram utilizados 15 casais de P. xylostella, totalizando 15 gaiolas para cada tratamento. Foram avaliados a fecundidade, a fertilidade e a viabilidade de ovos, além do IDO (Figura 2).

Foi realizado em delineamento experimental inteiramente casualizado (DIC) e as médias da fecundidade, fertilidade e viabilidade de ovos foram comparadas pelo teste de $t$ de Student $(\mathrm{p}<0,05)$.

O cálculo do IDO foi determinado pelo número de ovos do tratamento controle e pelo número de ovos do tratamento com extrato, com base na formula: IDO $=(\mathrm{C}-\mathrm{T}) /(\mathrm{C}+\mathrm{T})$ x $100 \mathrm{e}$ os valores obtidos para cada tratamento foram analisados em esquema fatorial ( 2 x 2) plantas e concentrações e as médias comparadas pelo teste Tukey $(\mathrm{p}<0,05)$. 
Figura 2. Esquema representativo dos bioensaios sem chance e com chance de escolha para oviposição de $P$. xylostella em discos de couve tratados. Bioensaios desenvolvidos em condições controladas de temperatura $\left(25 \pm 2{ }^{\circ} \mathrm{C}\right)$, umidade relativa $(55$ $\pm 5 \%$ ) e fotoperíodo (12 horas).

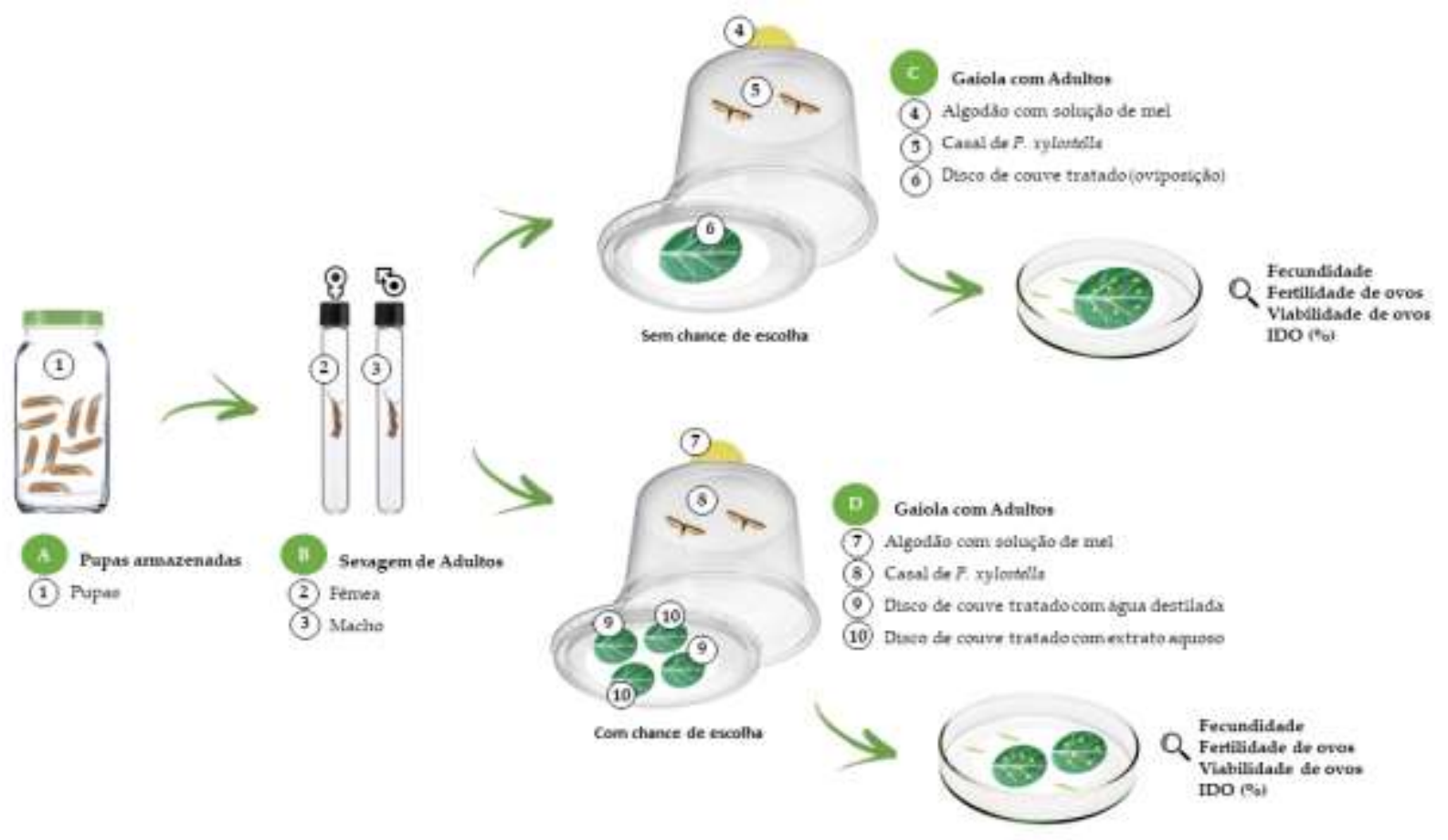

Fonte: Autores.

\subsection{Análise de dados}

A normalidade dos dados foi testada por Shapiro-Wilk e homogeneidade pelo teste de Bartlett, e quando necessário, foram transformados para $\sqrt{ } \mathrm{x}+0,5$ e arcoseno da $\sqrt{ } \mathrm{x} / 100$. A análise estatística foi realizada utilizando o software $\mathrm{R}$.

\section{Resultados}

\subsection{Teste de oviposição de $P$. xylostella sem chance de escolha}

Houve significância para o fator isolado planta para a fecundidade ( $\mathrm{F}=18,8797 ; \mathrm{GL}=2 ; p=0,0001)$, fertilidade dos $\operatorname{ovos}(\mathrm{F}=15,8683 ; \mathrm{GL}=2 ; p=0,0001) \mathrm{e}$ viabilidade dos ovos $(\mathrm{F}=27,8632 ; \mathrm{GL}=2 ; p<0,0001)$ (Tabela 1).

Houve significância para o fator isolado concentração para a fecundidade $(\mathrm{F}=4,6962 ; \mathrm{GL}=1 ; p=0,0330)$, fertilidade $\operatorname{dos} \operatorname{ovos}(\mathrm{F}=5,0925 ; \mathrm{GL}=1 ; p=0,0026)$ e viabilidade dos ovos $(\mathrm{F}=6,8737 ; \mathrm{GL}=1 ; p=0,0103)$ (Tabela 1).

Houve interação significativa entre os fatores (planta e concentração) apenas para o IDO (F = 4,938; GL = 1; $p=$ 0,02852) (Tabela 2).

O IDO foi superior a $35 \% \mathrm{em}$ todos os tratamentos avaliados. Notamos que o extrato da espécie $S$. erecta apresentou maior efeito dissuasor na concentração $10 \%(71,44 \pm 7,05)$ em relação a concentração $5 \%(35,61 \pm 11,13)$. A espécie $S$. marginata apresentou IDO semelhantes nas duas concentrações avaliadas (Tabela 2) e diferiu de S. erecta na concentração 5\%.

Para o fator isolado planta notamos que todos os parâmetros avaliados foram significativamente reduzidos nos tratamentos utilizando os extratos aquosos das duas espécies vegetais e que a concentração $10 \%$, no fator isolado concentração, obteve maior êxito na redução destas características (Tabela 1). 
Tabela 1. Médias de fecundidade, fertilidade dos ovos e viabilidade dos ovos para os fatores isolados planta ( $S$. erecta e $S$. marginata) e concentração ( $5 \%$ e $10 \%)$ no teste de oviposição sem chance de escolha $\left(25 \pm 2{ }^{\circ} \mathrm{C} ; 55 \pm 5\right.$ UR; $12 \mathrm{~h}$ fotofase).

\section{Fator Planta}

\begin{tabular}{lccc}
\hline & Fecundidade & Fertilidade dos ovos & Viabilidade dos ovos (\%) \\
\hline Controle & $162,40 \pm 10,45 \mathrm{a}$ & $106,33 \pm 8,29 \mathrm{a}$ & $54,08 \pm 2,61 \mathrm{a}$ \\
S. erecta & $69,93 \pm 12,69 \mathrm{~b}$ & $43,20 \pm 9,10 \mathrm{~b}$ & $21,85 \pm 3,96 \mathrm{~b}$ \\
S. marginata & $72,60 \pm 14,03 \mathrm{~b}$ & $44,26 \pm 10,53 \mathrm{~b}$ & $19,68 \pm 4,62 \mathrm{~b}$ \\
\hline
\end{tabular}

\begin{tabular}{lrrr}
\hline \multicolumn{3}{c}{ Fator Concentração } \\
\hline $5 \%$ & $116,80 \pm 11,75 \mathrm{a}$ & $76,42 \pm 9,04 \mathrm{a}$ & $37,39 \pm 3,90 \mathrm{a}$ \\
$10 \%$ & $86,48 \pm 11,86 \mathrm{~b}$ & $52,77 \pm 8,17 \mathrm{~b}$ & $26,34 \pm 3,72 \mathrm{~b}$
\end{tabular}

Médias ( \pm erro padrão) seguidas por letras distintas minúsculas nas colunas diferem entre si pelo teste de Tukey ao nível de significância a 5\% de probabilidade.

Fonte: Autores.

Tabela 2. Índice de Dissuasão de oviposição obtido em teste sem chance de escolha os tratamentos com extratos vegetais das espécies de $S$. erecta e $S$. marginata nas concentrações $5 \%$ e $10 \%\left(25 \pm 2{ }^{\circ} \mathrm{C} ; 55 \pm 5 \mathrm{UR}\right.$; $12 \mathrm{~h}$ fotofase $)$.

\begin{tabular}{cccc}
\hline \multirow{2}{*}{ Planta } & \multicolumn{3}{c}{ Índice de Dissuasão de oviposição (\%) } \\
\cline { 2 - 4 } & \multicolumn{3}{c}{ Concentrações } \\
\cline { 2 - 4 } & $55,61 \pm 11,13 \mathrm{bB}$ & $71,44 \pm 7,05 \mathrm{aA}$ & Classificação (IDO) \\
\hline S. erecta marginata & $68,70 \pm 4,22 \mathrm{aA}$ & $69,75 \pm 7,31 \mathrm{aA}$ & Dissuasor \\
\hline CV $(\%)$ & & 49,37 & Dissuasor \\
\hline
\end{tabular}

Médias ( \pm erro padrão) seguidas por letras minúsculas nas colunas comparam plantas na mesma concentração, enquanto. médias ( \pm erro padrão) seguidas por letras maiúsculas nas linhas comparam concentrações para a mesma espécie de planta. Letras distintas diferem entre si pelo teste de Tukey ao nível de significância a $5 \%$ de probabilidade. CV = Coeficiente de variação.

Fonte: Autores.

\subsection{Teste de oviposição de $P$. xylostella com chance de escolha}

A fecundidade foi reduzida em todos os tratamentos com extratos aquosos de $S$. erecta $5 \%(\mathrm{t}=4,609 ; \mathrm{GL}=1 ; p=$ $0,0002)$ e $10 \%(\mathrm{t}=6,4516 ; \mathrm{GL}=1 ; p=0,000001078)$ e $S$. marginata $5 \%(\mathrm{t}=5,17896 ; \mathrm{GL}=1 ; p=0,0001)$ e $10 \%(\mathrm{t}=3,7319 ;$ $\mathrm{GL}=1 ; p=0,0016$ ) (Tabela 3; Figura 3). De modo similar, a fertilidade dos ovos também foi significativamente menor nos discos tratados com extratos aquosos de $S$. erecta $5 \%(\mathrm{t}=3,5537 ; \mathrm{GL}=1 ; p=0,0026)$ e $10 \%(\mathrm{t}=5,5511 ; \mathrm{GL}=1 ; p=0,0001)$ e $S$. marginata $5 \%(\mathrm{t}=4,5684 ; \mathrm{GL}=1 ; p=0,0002)$ e $10 \%(\mathrm{t}=3,4793 ; \mathrm{GL}=1 ; p=0,0027)$ (Tabela 3; Figura 4). 
Figura 3. Média da fecundidade (número de ovos) $(p<0,05)$ de $P$. xylostella nos discos de couve foliares tratados com água destilada (controle) e extratos aquosos de Serjania spp. nas concentrações 5\% e $10 \%$ no teste de oviposição com chance de escolha. Médias comparadas pelo teste de t Student a 5\% de probabilidade.

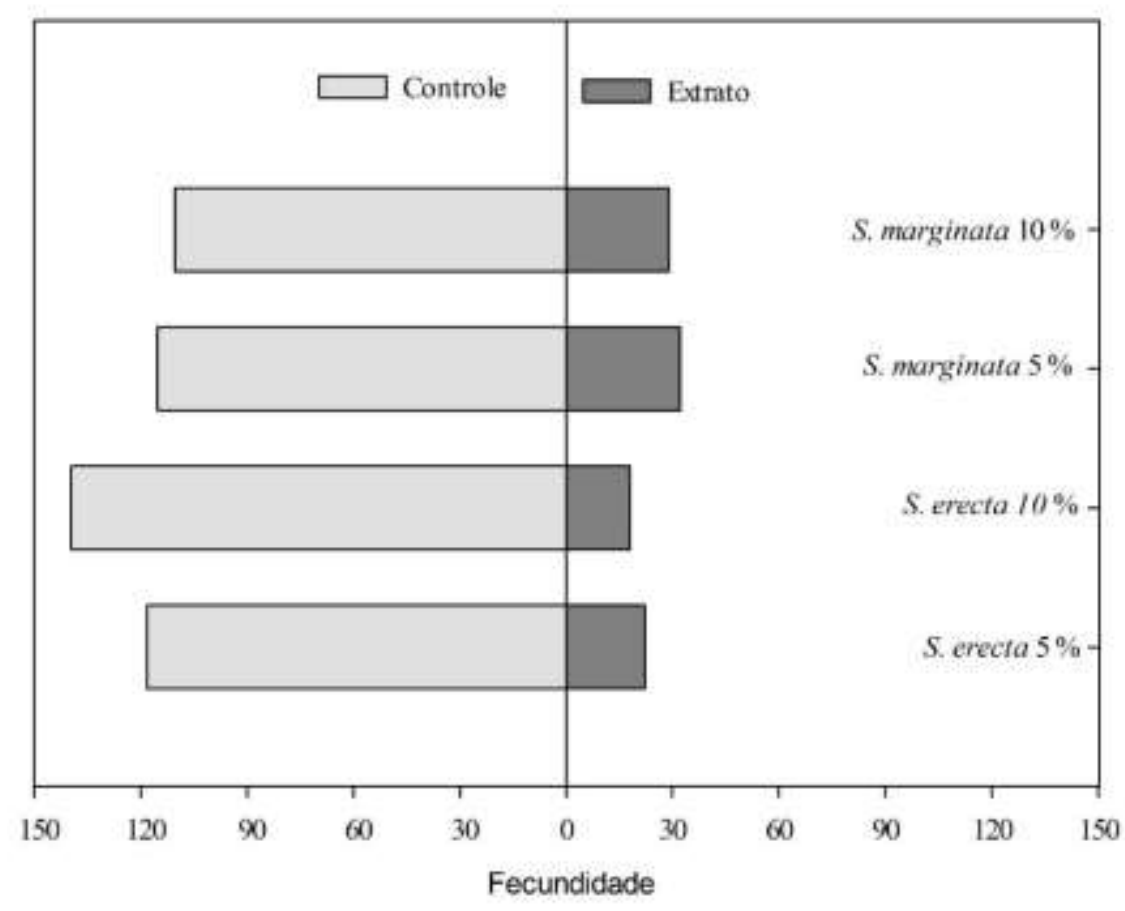

Fonte: Autores.

Figura 4. Média da fertilidade dos ovos (número de larvas recém-emergidas) de P. xylostella $(p<0,05)$ ovipositados nos discos de couve foliares tratados com água destilada (controle) e extratos aquosos de Serjania spp. nas concentrações 5\% e $10 \%$ no teste de oviposição com chance de escolha. Médias comparadas pelo teste t de Student a 5\% de probabilidade.

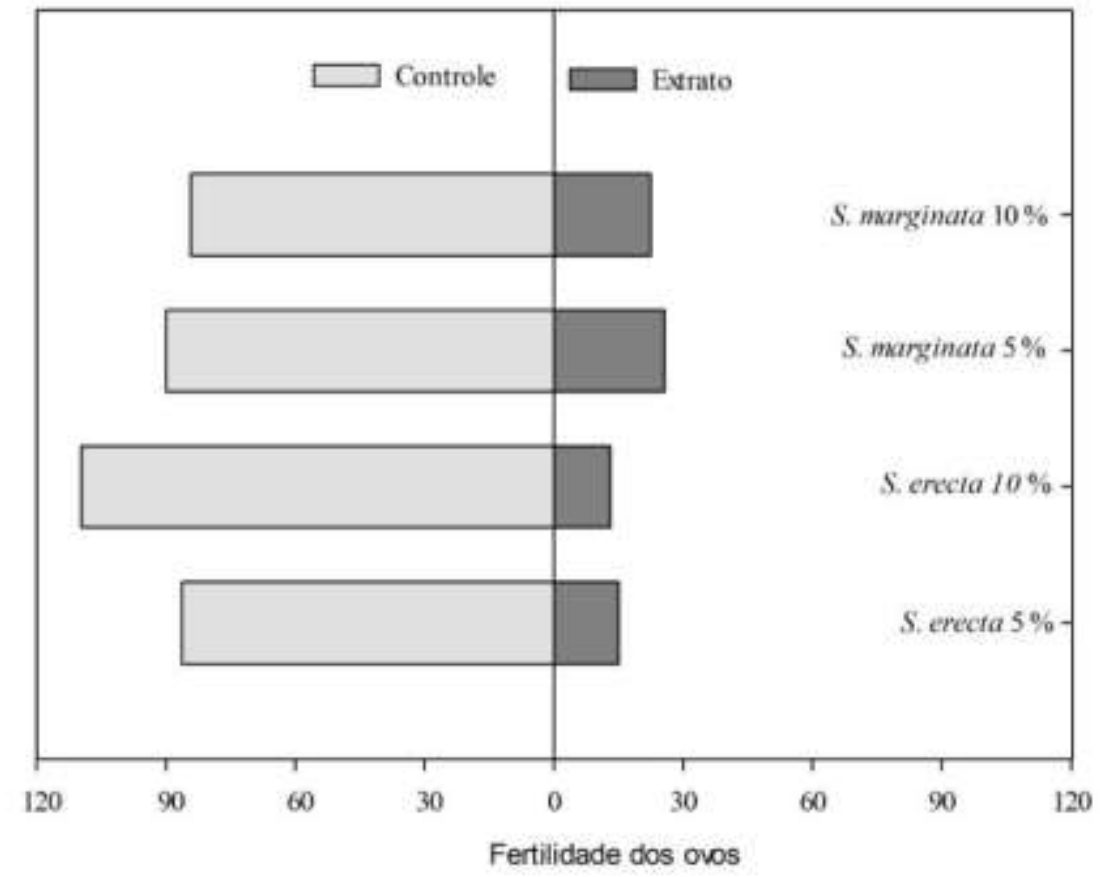

Fonte: Autores. 
A viabilidade de ovos foi significativamente reduzida nos tratamentos com extratos aquosos da espécie S. erecta $5 \%$ ( $\mathrm{t}$ $=2,6061 ; \mathrm{GL}=1 ; p=0,0158)$ e $10 \%(\mathrm{t}=4,1557 ; \mathrm{GL}=1 ; p=0,0005685)$, entretanto, para a espécie $S$. marginata, apenas o tratamento de concentração $10 \%(\mathrm{t}=2,446 ; \mathrm{GL}=1 ; p=0,0214)$ diminuiu a viabilidade dos ovos (Tabela 3 ).

O IDO apresentou resultados superiores a 55\% em todos os tratamentos, evidenciando o efeito dissuasor das espécies vegetais, independente da concentração utilizada para o teste com chance de escolha (Figura 5).

Tabela 3. Médias do controle e do extrato aquoso vegetal em cada tratamento para a fecundidade, fertilidade dos ovos e viabilidade de ovos no teste de oviposição com chance de escolha $\left(25 \pm 2^{\circ} \mathrm{C} ; 55 \pm 5\right.$ UR; $12 \mathrm{~h}$ fotofase $)$.

\begin{tabular}{lcccccc}
\hline \multirow{2}{*}{ Tratamentos } & \multicolumn{2}{c}{ Fecundidade } & \multicolumn{2}{c}{ Fertilidade dos ovos } & \multicolumn{2}{c}{ Viabilidade dos ovos (\%) } \\
\cline { 2 - 7 } & Controle & Extrato & Controle & Extrato & Controle & Extrato \\
\hline S. erecta 5\% & $118,06 \pm 19,83 \mathrm{~A}$ & $22,20 \pm 6,27 \mathrm{~B}$ & $86,33 \pm 19,52 \mathrm{~A}$ & $15,13 \pm 4,98 \mathrm{~B}$ & $42,80 \pm 8,02 \mathrm{~A}$ & $18,44 \pm 4,79 \mathrm{~B}$ \\
S. erecta 10\% & $139,40 \pm 18,49 \mathrm{~A}$ & $17,86 \pm 3,56 \mathrm{~B}$ & $109,66 \pm 17,15 \mathrm{~A}$ & $13,06 \pm 2,93 \mathrm{~B}$ & $52,08 \pm 7,72 \mathrm{~A}$ & $17,45 \pm 3,11 \mathrm{~B}$ \\
S. marginata 5\% & $115,26 \pm 15,08 \mathrm{~A}$ & $32,13 \pm 5,68 \mathrm{~B}$ & $90,00 \pm 13,12 \mathrm{~A}$ & $25,60 \pm 5,14 \mathrm{~B}$ & $44,56 \pm 5,86 \mathrm{~A}$ & $30,69 \pm 5,95 \mathrm{~A}$ \\
S. marginata 10\% & $110,33 \pm 20,57 \mathrm{~A}$ & $28,93 \pm 7,24 \mathrm{~B}$ & $84,26 \pm 16,77 \mathrm{~A}$ & $22,46 \pm 5,84 \mathrm{~B}$ & $42,07 \pm 6,81 \mathrm{~A}$ & $21,07 \pm 5,21 \mathrm{~B}$ \\
\hline
\end{tabular}

Médias ( \pm erro padrão) seguidas por letras maiúsculas distintas nas linhas diferem entre si pelo teste $\mathrm{t}$ de student a $5 \%$ de probabilidade. Fonte: Autores.

\section{Discussão}

O efeito de antixenose causado pelas espécies $S$. erecta e $S$. marginata foi observado em todos os tratamentos, independentemente do método (sem chance e com chance) utilizado, podendo ser decorrente da presença de compostos bioativos oriundos dos metabólitos secundários destas espécies vegetais (Guimarães et al., 2015; Moreira et al., 2019).

Em todos os tratamentos, notamos a redução da fecundidade que pode ser justificada, possivelmente, pela mudança comportamental das fêmeas de P. xylostella, quando em contato com os tratamentos contendo extratos de Serjania spp., pois geralmente, o processo de oviposição é estimulado pelos compostos glucosinolatos que são percebidos pelos quimiorreceptores de contato da mariposa, entretanto, os compostos inibidores oriundos dos extratos, podem sobrepor os glucosinolatos, interferindo no processo quimioreceptivo da P. xylostella e diminuindo a oviposição (Fahey et al., 2001; Feng et al., 2017).

Entre os compostos inibidores, os terpenos são constantemente relatados na literatura como substancias inibidoras de oviposição e atrativas para inimigos naturais (Qiu et al., 1998; Borges et al., 2018). O extrato botânico de espécies de Ludwigia reduziu a fecundidade e foram tóxicos para ovos e larvas de $P$. xylostella, com presença de taninos, flavonoides e compostos fenólicos que também podem ter influenciado na redução desta característica (Ferreira et al. 2020). Triagens fitoquímicas mostram que os extratos das espécies $S$. erecta e $S$. marginata apresentaram altos teores de taninos, saponinas, alcaloides, flavonoides e terpenos, que podem ter contribuído sinergicamente para redução da fecundidade de P. xylsotella (Gomig et al., 2008; Guimarães et al., 2015; Périco et al., 2015; Moreira et al., 2019).

Em estudo recente sobre a ação de $S$. marginata sobre $P$. xylostella foi constatado a redução da fecundidade nos tratamentos contendo extrato aquosos de S. marginata nas concentrações 5\% e 10\% e confirmaram a supressão da oviposição deste microlepidóptero, principalmente em concentrações maiores, sendo atribuído, possivelmente, à presença de compostos bioativos presentes nos extratos aquosos de folhas de S. marginata (Couto et al., 2019).

De acordo com os resultados, a fertilidade e a viabilidade dos ovos também diminuíram em quase todos os tratamentos utilizando extratos aquosos de S. erecta e S. marginata, principalmente na maior concentração (10\%), devido, possivelmente, ao 
contato direto dos ovos recém ovipositados sobre o substrato tratado e pela volatilização dos compostos oriundos dos extratos (Kumar et al., 2009; Rocha et al., 2021). As substâncias bioativas podem penetrar pelo córion dos ovos, através dos microcanais aerópilos que intermediam as trocas gasosas naturais do embrião com o meio externo, interferindo no processo de oxigenação do embrião, nos processos hormonais e bioquímicos, afetando diretamente o desenvolvimento do organismo (Fagoonee \& Lauge, 1981; Smith \& Salkeld, 1996). Substâncias bioativas como os flavonoides, saponinas e taninos podem reduzir significativamente a fecundidade de ovos, principalmente, quando utilizados sinergicamente, além disso, alguns compostos taninos podem se ligar a superfície do ovo, através de interações proteicas, interferindo no processo de oxigenação do embrião, comprometendo seu desenvolvimento e, consequentemente, causando alterações na fertilidade e viabilidade dos ovos (Engstrom et al., 2016).

A fertilidade e viabilidade dos ovos foram reduzidas, principalmente, utilizando extratos na concentração 10\%, pois maiores concentrações garantem maior vulnerabilidade de ovos aos compostos bioativo e podem afetar o desenvolvimento embrionário e a viabilidade dos ovos (Matharu \& Mehta, 2017; Sangha et al., 2017; Rocha et al., 2021). O efeito dissuasor ocorreu em todos os tratamentos, independentemente do método utilizado (sem chance ou com chance), entretanto, assim como nas demais características avaliadas, os maiores valores de IDO foram resultantes da maior concentração utilizada (10\%), em quase todos os tratamentos, pois a medida que a concentração aumenta espera-se a extração de mais substancias bioativas do material vegetal que podem contribuir para o aumento do efeito dissuasor (Torres et al., 2001; Silva et al., 2019).

Nos bioensaios realizados ambas espécies vegetais apresentaram resultados semelhantes, reduzindo a fecundidade, o número de larvas e a viabilidade dos ovos, pois estas espécies pertencem ao mesmo gênero botânico (Serjania) e, possivelmente, compartilham substâncias inseticidas e características ictiotóxicas semelhantes (Quintanilha \& Pinto, 2014; Andrade et al., 2015; Périco et al., 2015).

\section{Conclusão}

As espécies vegetais, S. erecta e S. marginata, apresentam capacidade de dissuadir a oviposição de $P$. xylostella, diminuindo a fecundidade, fertilidade e viabilidade de ovos, independentemente do método utilizado (sem chance ou com chance de escolha), entretanto, notamos que no método sem chance de escolha a concentração $10 \%$ apresentou melhores resultados. Assim, os extratos aquosos de S. erecta e $S$. marginata apresentam potencial para serem utilizados como bioinseticidas no controle de $P$. xyostella, principalmente na região local onde as espécies são nativas, promovendo o uso sustentável e econômico de métodos alternativos para o pequeno agricultor, minimizando as aplicações de pesticidas e os impactos ao meio ambiente.

\section{Agradecimentos}

Os autores agradecem o apoio e suporte da Universidade Federal da Grande Dourados, do Programa de Pós-Graduação de Entomologia e Conservação da Biodiversidade e da coordenação de Aperfeiçoamento de Pessoal de nível Superior (CAPES) pela bolsa concedida ao primeiro autor. Ao suporte financeiro, $\mathrm{n}^{\circ}$. 71/711.130/2018 concedido pela Fundação de Desenvolvimento, Educação, Ciência e Tecnologia de Matogrosso do Sul (FUNDECT).

\section{Referências}

Agboyi, L. K., Ketoh, G. K., Martin, T., Glitho, I. A., \& Tamo, M. (2016). Pesticide resistance in Plutella xylostella (Lepidoptera: Plutellidae) populations from Togo and Benin. International Journal of Tropical Insect Science, 36(4), 204-210. https://doi.org/10.1017/S1742758416000138

Amoabeng, B. W., Gurr, G. M., Gitau, C. W., Nicol, H. I., Munyakazi, L., \& Stevenson, P. C. (2013). Tri-Trophic insecticidal effects of African plants against cabbage pests. PLOS ONE, 8(4), e78651. https://doi.org/10.1371/journal.pone.0078651 
Andrade, J. N., Costa Neto, E. M., \& Brandão, H. (2015). Using ichthyotoxic plants as bioinsecticide: A literature review. Revista Brasileira de Plantas Medicinais, 17(4), 649-656. https://doi.org/10.1590/1983-084X/13_105

Arthropod Pesticide Resistance Database. (2021). Plutella xylostella. Acesso em 28 de julho, 2021 de https://www.pesticideresistance.org/

Barros, R., Thuler, R. T., \& Pereira, F. F. (2012). Técnica de criação de Plutella xylostella (L. 1758) (Lepidoptera: Yponomeutidae). Em D. Pratissoli (Org.), Técnicas de Criação de Pragas de Importância Agrícola, em Dietas Naturais (pp. 65-84). Vitória, ES: Edufes.

Borges, E. D. O., Martins, C. B., da Silva R. R., \& Zarbin, P. H. (2018). Terpenoids dominate the bouquet of volatile organic compounds produced by Passiflora edulis in response to herbivory by Heliconius erato phyllis (Lepidoptera: Nymphalidae). Arthropod-Plant Interactions, 12(1), 123-131. https://doi.org/10.1007/s11829-017-9560-2

Castillo-Sánchez, L. E., Jiménez, J. J., \& Delgado, M. A. (2010). Secondary metabolites of the Annonaceae, Solanaceae and Meliaceae families used as biological control of insects. Tropical and subtropical Agroecosystems, 12(1), 445-462.

Couto, I. F. S., Verza, S., Valente, F. I., Senna, B., Souza, S. A., Mauad, M., \& Mussury, R. M. (2019) Botanical extracts of the brazilian savannah affect feeding and oviposition of Plutella xylostella (Linnaeus, 1758) (Lepidoptera: Plutellidae). Journal of Agricultural Science, 11(5), 322-333. https://doi.org/10.1007/s10343-020-00520-8

Couto, I. F. S., Souza, S. A., Valente, F. I., Silva, R. M., Scalon, S. D. P. Q., Pereira, F. F., Silva, S. V., Carvalho, E. M., \& Mussury, R. M. (2020). Changes in the biological characteristics of Plutella xylostella using ethanolic plant extracts. Gesunde Pflanzen, 72(4), 383-391. https://doi.org/10.1007/s10343-020-005208

Engstrom, M. T., Karonen, M., Ahern, J. R., Baert, N., Payré, B., Hoste, H., \& Salminen, J. P. (2016). Chemical structures of plant hydrolyzable tannins reveal their in vitro activity against egg hatching and motility of Haemonchus contortus nematodes. Journal of agricultural and food chemistry, 64(4), 840-851. https://doi.org/10.1021/acs.jafc.5b05691

Fagoonee, I., \& Lauge, G. (1981). Noxious effects of neem extract of Crocidolomia binotalis. Phytoparasitica, 9(2), 111-118. https://doi.org/10.1007/BF03158453

Fahey, J. W., Zalcmann, A. T., \& Talalay, P. (2011). The chemical diversity and distribution of glucosinolates and isothiocyanates among plants. Phytochemistry, 56(1), 5-51. https://doi.org/10.1016/S0031-9422(00)00316-2

Feng, B., Qian, K., \& Du, Y. J. (2017). Floral volatiles from Vigna unguiculata are olfactory and gustatory stimulants for oviposition by the bean pod borer moth Maruca vitrata. Insects, 8(2), 60. https://doi.org/10.3390/insects8020060

Ferreira, E. A., de Souza, S. A., Domingues, A., Da Silva, M. M. M., Padial, I. M. P. M., de Carvalho, E. M., Cardoso, C. A. L., da Silva, S. V., \& Mussury, R. M. (2020). Phytochemical screening and bioactivity of Ludwigia spp. in the control of Plutella xylostella (Lepidoptera: Plutellidae). Insects, 11(9), 596. https://doi.org/10.3390/insects 11090596

Fonseca, J., Couto, I. F. S., Silva, R. M., Fioratti, C. A. G., Pereira, F. F., Mauad, M., Scalon, S. P. Q., Carvalho, E. M., \& Mussury, R. M. (2018). Efeito de extratos metanólicos de Stryphnodendron adstringens (Mart) coville na alimentação e reprodução de Plutella xylostella L. (Lepidoptera: Plutellidae). Interciência, 43(3),182-187.

Furlong, M. J., Zu-Hua, S., Yin-Quan, L., Shi-Jian, G., Yao-Bin, L., Shu-Sheng, L., \& Zalucki, M. P. (2004). Experimental analysis of the influence of pest management practice on the efficacy of an endemic arthropod natural enemy complex of the diamondback moth. Journal of Economic Entomology, 97(6), 1814 27. https://doi.org/10.1093/jee/97.6.1814

Iannacone, J., Onofre, R., Huanqui, O., Giraldo, J., Mamani, N., Miglio, M. C., \& Alvariño, L. (2007). Evaluación del riego ambiental del insecticida metamidofos en bioensayos con cuatro organismos acuáticos no Destinatarios. Agricultura Técnica, 67(2), 126-138. http://dx.doi.org/10.4067/S036528072007000200002

Iannacone, J., Alvariño, L., Murrugarra, Y., Arrascue, A., Alayo, M., \& Salazar, N. (2008). Selectividad del insecticida metamidofos en ocho organismos terrestres no destinatarios. Journal of Brazilian Society of Ecotoxicology, 3(1):23-34. DOI:10.5132/jbse.2008.01.004

Krinski, D., Massaroli, A., \& Machado, M. (2014). Potencial inseticida de plantas da família Annonaceae. Revista Brasileira de Fruticultura, 36, $225-242$. https://doi.org/10.1590/S0100-29452014000500027

Kumar, R., Sharma, K. C., \& Kumar, D. (2009). Studies on ovicidal effects of some plant extracts against the diamondback moth, Plutella xylostella (L.) infesting cauliflower crop. Biological Forum-An International Journal, 1(1), 47-50.

Li, Z. Y., Zalucki, M. P., Bao, H. L., Chen, H. Y, Hu, Z. D., \& Feng, X. (2012). Population dynamics and 'outbreaks' of diamondback moth, Plutella xylostella, in Guangdong Province, China: climate or the failure of management? Journal of Economic Entomology, 105(3), 739-52. https://doi.org/10.1603/EC11384

Loiseleur, O. (2017). Natural products in the discovery of agrochemicals. CHIMIA International Journal for Chemistry, 71(12), 810-822. https://doi.org/10.2533/chimia.2017.810

Gomig, F., Pietrovski, E. F., Guedes, A., Dalmarco, E. M., Calderari, M. T., Guimarães, C. L., Pinheiro, R. M., Cabrini, D. A., \& Otuki, M. F. (2008). Topical anti-inflammatory activity of Serjania erecta Radlk (Sapindaceae) extracts. Journal of Ethnopharmacology, 118(2), 220-224. https://doi.org/10.1016/j.jep.2008.03.017

Guimarães, C. C., Oliveira, D. D., Valdevite, M., Saltoratto, A. L. F., Pereira, S. I. V., França, S. C., Pereira, A. M. S., \& Pereira, P. S. (2015). The glycosylated flavonoids vitexin, isovitexin, and quercetrin isolated from Serjania erecta Radlk (Sapindaceae) leaves protect PC12 cells against amyloid- $\beta 25-35$ peptideinduced toxicity. Food and Chemical Toxicology, 86, 88-94. https://doi.org/10.1016/j.fct.2015.09.002 
Huang, X., \& Renwick, J. A. A. (1993). Differential selection of host plants by two Pieris species: the role of oviposition stimulants and deterrents. Entomologia experimentalis et applicata, 68(1), 59-69. https://doi.org/10.1111/j.1570-7458.1993.tb01689.x

Isman, M. B., \& Grienesen, M. L. (2014) Botanical inseticticide research: many publications, limited useful data. Triends in Plant Science, 19(3), 140-145. https://doi.org/10.1016/j.tplants.2013.11.005

Krinski, D., Massaroli, A., \& Machado, M. (2014). Potencial inseticida de plantas da família Annonaceae. Revista Brasileira de Fruticultura, 36, 225-242. https://doi.org/10.1590/S0100-29452014000500027

Lundgren, L. (1975). Natural plant chemicals acting as ovipsition deterrents on cabbage butterflies (Pieris brassicae (L.), P. rapae (L.) and P. napi (L)). Zoologica Scripta, 4(1), 253-258.

Marchioro, C. A., \& Foerster, L. A. (2011). Development and survival of the diamondback moth, Plutella xylostella (L.) (Lepidoptera: Yponomeutidae) as a function of temperature: effect on the number of generations in tropical and subtropical regions. Neotropical Entomology, 40(5), 533-541. https://doi.org/10.1590/S1519-566X2011000500003

Massarolli, A., Bersani, B. C. G., Roza, F. H. D., Butnariu, A. R., Pereira, M. J. B., \& Foerster, L. A. (2019). Evaluation of Leaf Consumption and Determination of Economic Injury Level Caused by Plutella xylostella (L.) (Lepidoptera: Plutellidae) in Brassica oleracea var. acephala (Brassicales: Brassicaceae). Journal of economic entomology, 112(4), 1805-1811. https://doi.org/10.1093/jee/toz113

Matharu, K, \& Mehta, P. (2017). Ovicidal activity of crude extracts of indigenous plant species against Plutella xylostella (L.) (Lepidoptera: Plutellidae). Environment \& Ecology, 35(1A), 285-289.

Mordue, A. J, \& Blackwell, A. (1993). Azadirachtin: an update. Journal of Insect Physiology, 39(11), 903-924. https://doi.org/10.1016/0022-1910(93)90001-8

Moreira, S. S., Tamashiro, L. K., Jorge, B. C., Balin, P. S., Heredia-Vieira, S. C., Almeida, G. L., Cardoso, C. A. L., Kassuya, C. A. L., \& Arena, A. C. (2019). Toxicological safety evaluation in acute and 28-day studies of aqueous extract from Serjania marginata Casar (Sapindaceae) leaves in rats. Journal of ethnopharmacology 231, 197-204. https://doi.org/10.1016/j.jep.2018.11.024

Pavela, R. (2016). History, presence and perspective of using plant extracts as commercial botanical insecticides and farm products for protection against insects - a review. Plant Protection Science, 52(4), 229-241. https://doi.org/10.17221/31/2016-PPS

Peres, L. L. S., Sobreiro, A. I., Couto, I. F. S., Silva, R. M., Pereira, F. F., Heredia-Vieira, S. C., Cardoso, C. A. L., Mauad, M., Scalon, S. P. Q., Verza, S. S., \& Mussury, R. M. (2017). Chemical compounds and bioactivity of aqueous extracts of Alibertia spp. in the control of Plutella xylostella L. (Lepidoptera: Plutellidae). Insects, 8(4), 125. https://doi.org/10.3390/insects8040125

Périco, L. L., Heredia-Vieira, S. C., Beserra, F. P., dos Santos, R. C., Weiss, M. B., Resende, F. A., Ramos, M. A. S., Bonifácio, B. V., Bauab, T. M., Varanda, E. A., Gobbi, J. I. F., da Rocha, L. R. M., Vilegas, W., \& Hiruma-Lima, C. A. (2015). Does the gastroprotective action of a medicinal plant ensure healing effects? An integrative study of the biological effects of Serjania marginata Casar. (Sapindaceae) in rats. Journal of Ethnopharmacology, 172, 312-324. https://doi.org/10.1016/j.jep.2015.06.025

Qiu, Y. T., van Loon, J. J., \& Roessingh, P. (1998). Chemoreception of oviposition inhibiting terpenoids in the diamondback moth Plutella xylostella. Entomologia experimentalis et applicata, 87(2), 143-155. https://doi.org/10.1046/j.1570-7458.1998.00316.x

Quintanilla, R. H. R., \& Pinto, A. C. (2014). Constituintes Químicos e Propriedades Biológicas de Espécies do Gênero Serjania. Revista Virtual de Química, 6(6), 1583-1606. doi:10.5935/RVQ.V6I6.772

Ramírez-Zamora, J., Salinas-Sánchez, D. O., Figueroa-Brito, R., Ramos-López, M. Á., Castañeda-Espinoza, J. D., \& Flores-Macías, A. (2020). Botanical extracts from Dodonaea viscosa (Sapindales: Sapindaceae) reduce hemocyte counts from Spodoptera exigua (Lepidoptera: Noctuidae) with potential insecticidal synergism with Isaria fumosorosea (Hypocreales: Cordycipitaceae). Biocontrol Science and Technology, 30(12), 1365-1376. https://doi.org/10.1080/09583157.2020.1826903

Rocha, A. N., de Carvalho, E. M., Mauad, J. R. C., \& Mussury, R. M. (2021). Tradescantia pallida L.(Commelinaceae) influences the activity of oviposition and feeding of Plutella xylostella (Lepidoptera: Plutellidae). Research, Society and Development, 10(8), e57610817583-e57610817583. https://doi.org/10.33448/rsd-v10i8.17583

Sangha, J. S., Astatkie, T., \& Cutler, G. C. (2017). Ovicidal, larvicidal, and behavioural effects of some plant essential oils on diamondback moth (Lepidoptera: Plutellidae). The Canadian Entomologist, 149(5), 639-648. https://doi.org/10.4039/tce.2017.13

Salinas-Sánchez, D. O., Avilés-Montes, D., Aldana-Llanos, L., Gutiérrez-Ochoa, M., Figueroa-Brito, R., \& Sotelo-Leyva, C. (2020). Efecto insecticida de Serjania schiedeana para el control de Spodoptera frugiperda bajo condiciones de laboratorio e invernadero. Southwestern Entomologist, 45(2), 521-530. https://doi.org/10.3958/059.045.0220

Schmutterer, H. (1988). Potential of azadirachtin-containing pesticides for integrated pest control in developing and industrialized countries. Journal of Insect Physiology, 34(7), 713-719. https://doi.org/10.1016/0022-1910(88)90082-0

Silva, P. R. C., Camaroti, J. R. S. L., Almeida, W. A., Ferreira, E. C. B., Paiva, P. M. G., Barros, R., Napoleão, T. H., \& Pontual, E. V. (2019). Schinus terebinthifolia leaf extract is a larvicidal, pupicidal, and oviposition deterring agent against Plutella xylostella. South African Journal of Botany, 127, 124-128. https://doi.org/10.1016/j.sajb.2019.08.054

Smith, E. H., \& Salkeld, E. H. (1966). The use and action of ovicides. Annual Review of Entomology, 11(1), 331-368. https://doi.org/10.1146/annurev.en.11.010166.001555 
Research, Society and Development, v. 10, n. 14, e198101421802, 2021

(CC BY 4.0) | ISSN 2525-3409 | DOI: http://dx.doi.org/10.33448/rsd-v10i14.21802

Souza, A. S., Couto, I. F. S., Silva, M. P., Cardoso, C. A. L., Scalon, S. P. Q., Pereira, F. F., Carvalho, E. M., \& Mussury, R. M. (2019). Aqueous extracts of species of the genus Campomanesia (Myrtaceae) affect biological characteristics of Plutella xylostella (Linnaeus, 1758) Lepidoptera: Plutellidae. Journal of Agricultural Science, v. 11(5), 1916-9760. Doi: 10.5539/jas.v11n5p334

Tagliari, M. S., Knaak, N., \& Fiuza, L. M. (2004). Plantas inseticidas: interações e compostos. Pesquisa Agropecuária Gaúcha, 10(1/5):101-111.

Torres, A. L., Barros, R., \& Oliveira, J. V. (2001). Efeito de extratos aquosos de plantas no desenvolvimento de Plutella xylostella (L.) (Lepidoptera: Plutellidae). Neotropical Entomology, 30(1), 151-156. https://doi.org/10.1590/S1519-566X2001000100022

Ulmer, B., Gillotta, C., Woods, D., \& Erlandson, M. (2002). Diamondback moth, Plutella xylostella (L.), feeding and oviposition preferences on glossy and waxy Brassica rapa (L.) lines. Crop Protection, 21(4), 327-331. https://doi.org/10.1016/S0261-2194(02)00014-5

Zalucki, M. P., Shabbir, A., Silva, R., Adamson, D., Shu-Sheng, L., \& Furlong, M. J. (2012). Estimating the economic cost of one of the world's major insect pests, Plutella xylostella (Lepidoptera: Plutellidae): just how long is a piece of string? Journal of Economic Entomology, 105(4), 1115- 1129. https://doi.org/10.1603/EC12107

Zhang, S., Zhang, X., Shen, J., Mao, K., You, H., \& Li, J. (2016). Susceptibility of field populations of the diamondback moth, Plutella xylostella, to a selection of insecticides in Central China. Pesticide Biochemistry Physiology, v. 132, 38-46. https://doi.org/10.1016/j.pestbp.2016.01.007 